\title{
UNCERTAINTY CHARACTERIZATION FRAMEWORK FOR STEADY-STATE AND TRANSIENT NEUTRONICS SIMULATIONS OF A CANDU REACTOR
}

\author{
Hany S. Abdel-Khalik ${ }^{1}$, Alexandre Trottier ${ }^{2}$, Dumitru Serghiuta ${ }^{3}$, and Dongli Huang ${ }^{1}$ \\ ${ }^{1}$ Purdue University, School of Nuclear Engineering \\ 205 Gates Rd., West Lafayette IN 47907 \\ ${ }^{2}$ Canadian Nuclear Laboratories \\ 1 Plant Road, Chalk River, Ontario, Canada, K0J 1P0 \\ ${ }^{3}$ Canadian Nuclear Safety Commission \\ 280 Slater, Ottawa, Ontario, K1P 5S9
}

abdelkhalik@purdue.edu, alexandre.trottier@cnl.ca,dumitru.serghiuta@canada.ca, huang714@purdue.edu

\begin{abstract}
This paper reports on the development and testing of a comprehensive few-group cross section input uncertainty library for the NESTLE-C nodal diffusion-based nuclear reactor core simulator. This library represents the first milestone of a first-of-a-kind framework for the integrated characterization of uncertainties in steady-state and transient CANDU reactor simulations. The objective of this framework is to propagate, prioritize and devise a mapping capability for uncertainties in support of model validation of best-estimate calculations. A complete framework would factor both input and modeling uncertainty contributions. The scope of the present work is limited to the propagation of multi-group cross-section uncertainties through lattice physics calculations down to the few-group format, representing the input to the NESTLE-C core simulator, and finally to core responses of interest.
\end{abstract}

KEYWORDS: uncertainty characterization, CANDU neutronics core simulation, reduced order modeling.

\section{INTRODUCTION}

Traditionally, uncertainties in reactor calculations were addressed using a conservative bounding analysis. More recently, the best estimate plus uncertainty (BEPU) method has been sought as a more realistic alternative to the conservative bounding analysis, contingent upon the proper quantification of uncertainties of best-estimate (BE) simulation results. To fully realize the benefits of the BEPU method, one must explicitly account for all sources of uncertainties, including initial and boundary conditions, modeling and numerical sources of uncertainties, as well as those originating from the individual codes used in the overall coupled simulation.

The Canadian Nuclear Safety Commission (CNSC) initiated a multi-phase research project to evaluate and implement an integrated Uncertainty Characterization Framework (UCF) with primary application to CANDU neutronics calculations $[1,2,3]$ to enhance the capability for independent review of safety cases based on realistic methodologies which rely on complex 3D-neutronics/thermal-hydraulic coupled simulations. 
The adopted UCF involves three key processes. The first process, uncertainty quantification (UQ), propagates all known sources of simulation uncertainties to understand their impact on the reactor behavior. The second, sensitivity analysis (SA), acts in conjunction with UQ to identify key sources of uncertainties. The third, uncertainty mapping (UM), predicts realistic responses and uncertainties for a system based on the combined use of UQ, SA, and experimental results.

The focus of this paper is on the UQ process. Its scope is limited to the propagation of nuclear data uncertainties starting with a standard multi-group energy structure down to three different few-group energy structures and ultimately to the full core stand-alone neutronics simulations. To ensure computational feasibility, the framework is designed to employ well-proven approaches for reduced order modeling $(\mathrm{ROM})$, previously applied to light water reactor (LWR) calculations $[4,5]$.

Modeling uncertainties were minimized by employing a continuous energy Monte Carlo transport model based on SERPENT [6,7] to generate the reference few-group parameters for core-wide calculations in different group formats, including two-, four-, and eight-group structures. The sensitivity of the propagated uncertainties is measured by repeating the UQ analysis with different modeling assumptions, e.g., collapsing to different few-group structure, employing deterministic methods to generate the few-group parameters, etc. This approach is justified during this process as it provides an initial estimate of the impact of modeling uncertainties on the propagated few-group parameters uncertainties.

The focus of this paper is on the development of an uncertainty library for use with NESTLE-C [8] a multigroup neutron diffusion simulation code used for pressurized heavy water reactor simulations. A utility was developed to automate the process of generating statistically consistent random samples for the NESTLE-C cross section input files using the developed uncertainty library, executing NESTLE-C cases, and collating the results.

\section{UNCERTAINTY PROPAGATION ON CANDU CORE SIMULATION IN TWO GROUPS}

The uncertainty library was developed using the NEWT[9] and SAMPLER[10] codes. NEWT is a multigroup deterministic transport code used to compute group constants from repeated geometries in reactor core problems. SAMPLER is used to generate different samples of multi-group cross section input data to NEWT, based on covariance data encoded in nuclear evaluation data files. Using the specifications of the SERPENT models, NEWT models of a CANDU lattice cell were prepared for each of the three energy group partitions, i.e., the two-, four- and eight-group energy structures. In each case, a large set of independent simulations were performed and the homogenized cross sections were computed. Each simulation used a different sample nuclear cross section input data file provided by SAMPLER. The resulting two-group cross section input data were compared to SERPENT- and HELIOS-based data [11]. By employing both deterministic and Monte Carlo models to estimate the reference few-group parameters, the impact of some modeling uncertainties can be initially assessed on the propagated uncertainties. Similarly, by employing different group representations and resonance treatments ino NEWT, the impact of these modeling approximations can be studied. To understand the impact of the mean values of the cross sections, the uncertainty library was initially developed using HELIOS-based reference cross sections, and subsequently applied to the different SERPENT-based reference cross sections.

Initial tests were performed using a generic Natural Uranium-fueled CANDU-6 model implemented in NESTLE-C. Key parameters are listed in Table 1. The calculated parameters of interest are k-eff and the channel power distribution. The uncertainty library is implemented as reference cross section input file and $N$ independent cross section input files, each representing a potential variation of the cross section data about the mean. The parallel execution of $N+1$ NESTLE-C input files allowed for the generation of the full PDFs of core responses of interest. These additional files can also be generated in a deterministic manner, assuming that core responses are linearly correlated with variations in the few-group parameters, which 
allows for the direct calculation of the mean values and covariance matrix for core responses of interest, [12].

In regard to the computational procedure for the uncertainties, the few-group parameter uncertainty space is very large because as it contains cross-sections at all conditions required for proper cross-section functionalization. This includes all burnup steps (in the order of several tens) to get the cross-section dependence on burnup, all branch cases evaluated at each burnup step (in the order of few tens per burnup step) to calculate cross-section corrections due to changing local conditions such as fuel temperature and coolant temperature, and device cross-sections to calculate corrections resulting from insertion of reactivity devices. The matrix $\mathbf{C}_{F G}$ will thus be extremely large if calculated directly using a brute force method. Instead, we rely on a ROM approach, which constructs a compressed representation for the $\mathbf{C}_{F G}$ as:

$$
\begin{aligned}
\mathfrak{R}^{m \times N^{\prime}} \ni \mathbf{X}_{\sigma} & =\left[\begin{array}{llll}
\sigma_{1} & \sigma_{2} & \ldots & \sigma_{N^{\prime}}
\end{array}\right]=\mathbf{U S V}^{T}, \mathbf{U} \in \mathfrak{R}^{m \times r}, \mathbf{S} \in \mathfrak{R}^{r \times r} \\
\mathbf{C}_{F G} & =(\mathbf{U} \boldsymbol{\Sigma})(\mathbf{U} \boldsymbol{\Sigma})^{T}, \operatorname{diag}(\boldsymbol{\Sigma})=\frac{1}{\sqrt{N^{\prime}-1}} \operatorname{diag}(\mathbf{S})
\end{aligned}
$$

This approach requires an initial singular value decomposition (SVD) of the matrix containing the $N^{\prime}$ realizations -- a prime superscript is used to distinguish the number of runs employed to execute the lattice physics code, i.e., NEWT, from the number of executions of the NESTLE-C code -- of the $m$ fewgroup parameters, denoted by $\mathbf{X}_{\sigma}$, which determines the effective rank of the $\mathbf{C}_{F G}$ matrix. The $\mathbf{C}_{F G}$ matrix is represented in a compressed form from $m \sim 10^{4}$ for a typical CANDU reactor, down to $r$ which is in order of few tens. Figure 1 demonstrates how the few-group uncertainty space can be compressed using 40 singular vectors. The horizontal axis lists the number of singular vectors used in the approximation. The vertical axis lists the reconstruction error, in terms of the loss in coverage of the total variance of the cross section input domain. As the number of singular vectors is increased, the total variance of the input domain is recovered asymptotically. With 40 singular vectors, a reconstruction accuracy of $0.01 \%$ is achieved. The ROM-based uncertainty propagation and evaluation of reconstruction error bounds can be found in previous work $[13,14]$.

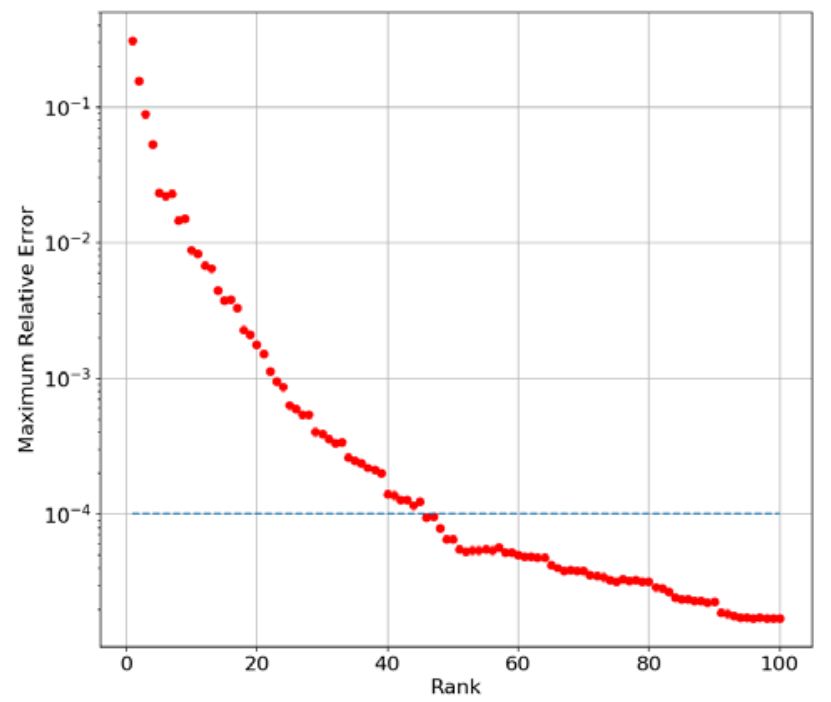

Figure 1. Reconstruction Errors of ROM-based Few-Group Cross-Section Uncertainty.

In Figure 2 the reference channel power distribution is presented, based on NESTLE-C calculations using HELIOS-based two-group cross section input data. Representative results of ROM-based uncertainty propagation to the steady-state core models using two-group structure are shown in Figure 3 (a) and (b) 
representing, respectively, the HELIOS-based and SERPENT-based reference cross-sections. Uncertainties of core channel power are higher in the center and at the inner bottom of the core, with the maximum relative uncertainty of $1 \%$. The power uncertainty distributions with different reference few-group input files are similar, while differences exist at several points. Figure 4 (a) and (b) show the power distribution discrepancies between NEWT-based and SERPENT-based reference few-group parameters using twogroup (2G) and four-group (4G) structures respectively. Results indicate that the relative power discrepancies with different reference values can be up to $3 \%$, which are non-negligible compared to the uncertainty values. This observation is one motivation to eventually extend the UCF to include modelingbased contributions.

\begin{tabular}{|r|l|}
\hline Parameter & Dimension \\
\hline Lattice pitch (square) & $28.575 \mathrm{~cm}$ \\
\hline Length of bundle & $49.53 \mathrm{~cm}$ \\
\hline Core length & $594.36 \mathrm{~cm}$ \\
\hline Core radius & $379.7 \mathrm{~cm}$ \\
\hline Channel count & 380 \\
\hline Fuel type & 12 \\
\hline Fuel bundles per channel & Natural Uranium \\
\hline Fuel bundle type & $37-$ element \\
\hline Heavy Water Moderator Purity & $99.97 \mathrm{wt} \% \mathrm{D}_{2} \mathrm{O}$ \\
\hline Heavy Water Coolant Purity & $99.20 \mathrm{wt} \% \mathrm{D}_{2} \mathrm{O}$ \\
\hline \# LZCs & 14 \\
\hline \# SORs & 28 \\
\hline \# ARs & 21 \\
\hline &
\end{tabular}

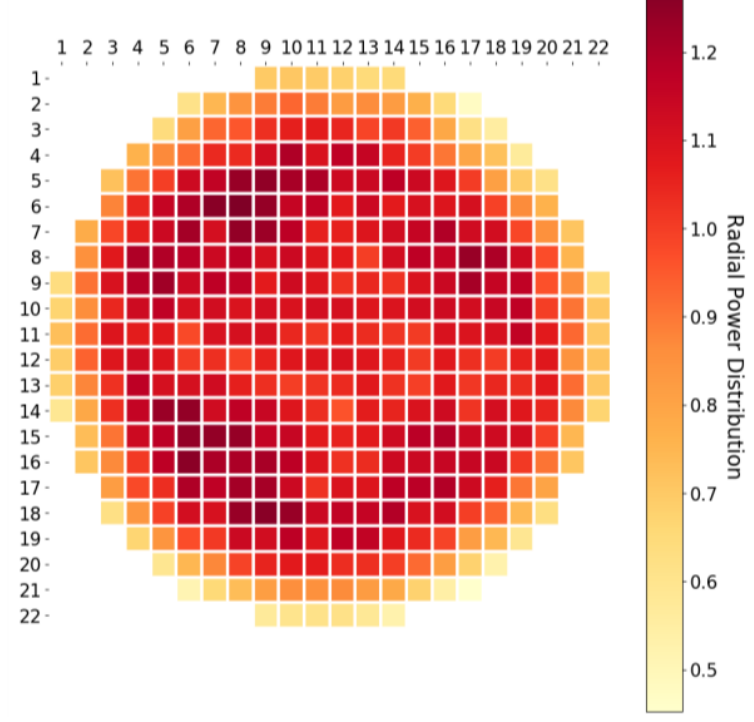

Table 1 Core Configurations

Figure 2. Channel Power Distribution.

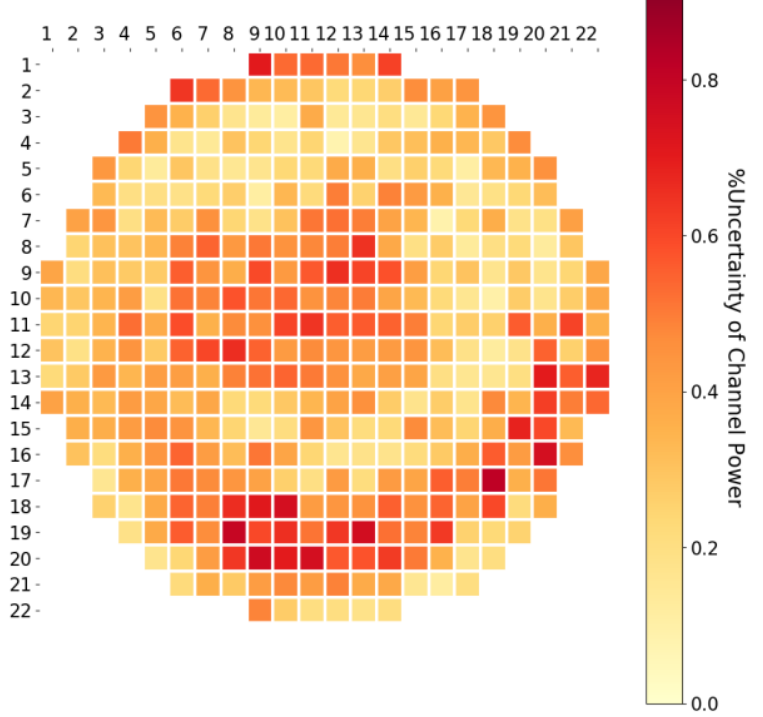

(a) HELIOS-based

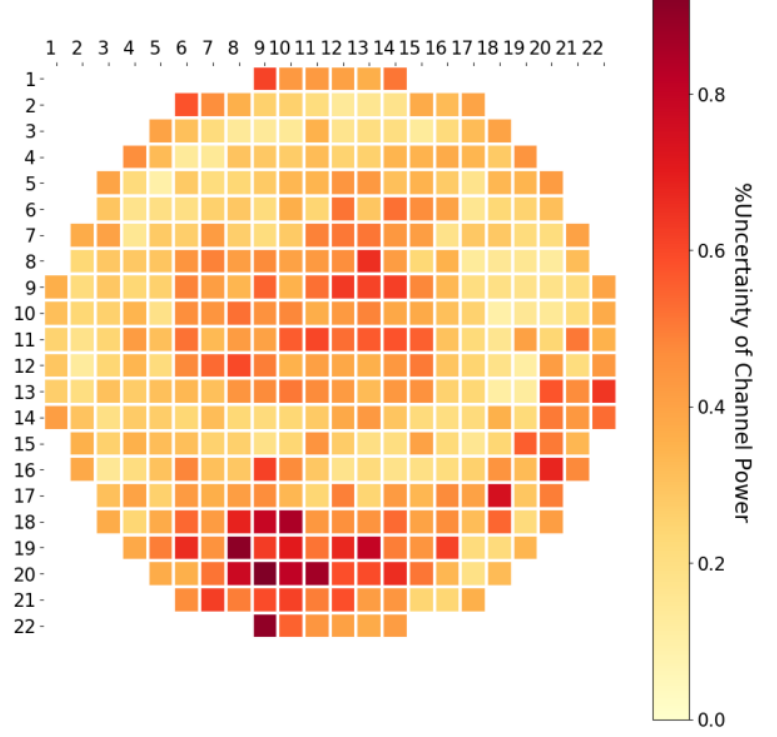

(b) SERPENT-based 
Figure 3. NESTLE-C Reference Channel Power Uncertainty with Two-Group Structure.

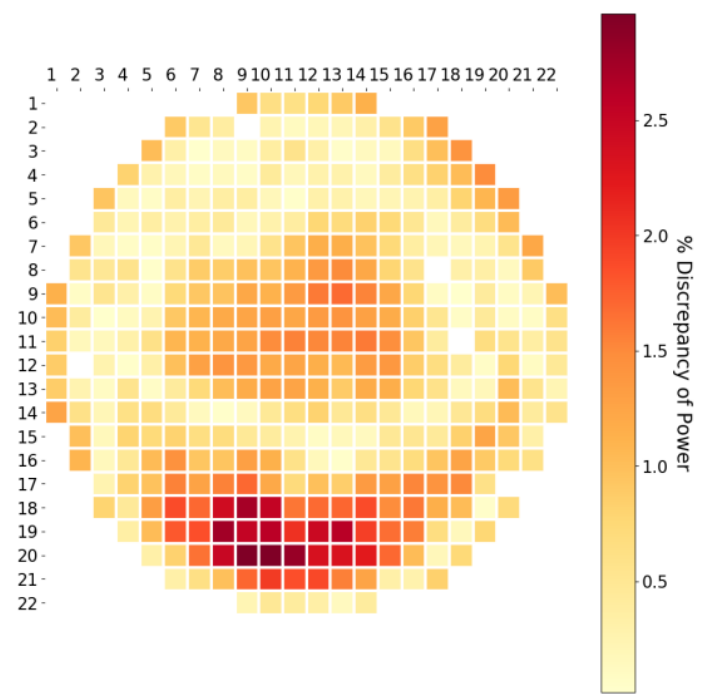

(a) NEWT_2G vs. SERPENT_2G

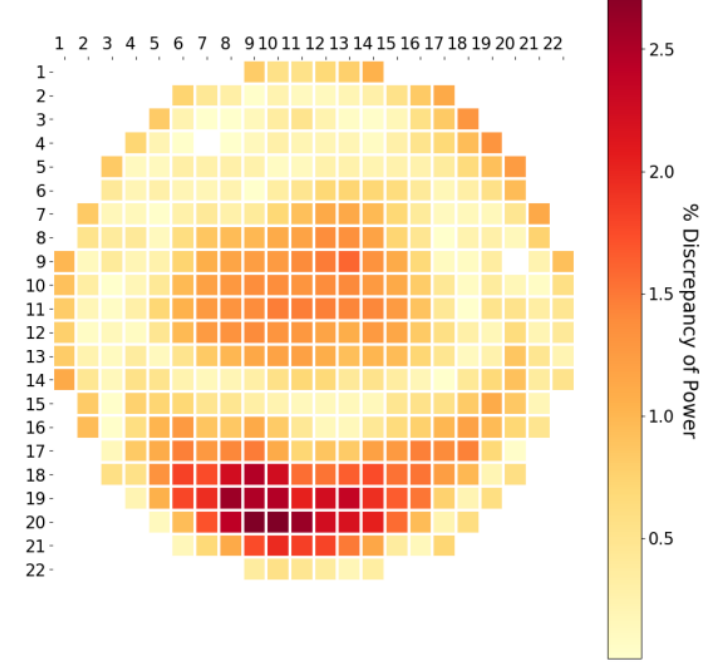

(b) NEWT_4G vs. SERPENT_4G

Figure 4. Percentage Discrepancy of NESTLE-C Calculated Reference Channel Power.

A generic loss of coolant accident (LOCA) model with fixed time-dependent thermal hydraulic conditions was used to test the impact of cross section uncertainties in transient cases. One thousand independent NESTLE-C sample cross section input files were prepared using the uncertainty library and the HELIOSbased two-group reference library. Each case was executed and the core relative power evolutions are depicted in Figure 5. The reference solution is traced in green, while a light blue envelop displays the uncertainty band. The yellow lines represent the density of all samples. The few-group parameter uncertainties result in a maximum core power uncertainty of $15 \%$ around $0.9 \mathrm{~s}$ when the core power reaches a maximum. Figure 6 contains the histograms of core relative power from the 1000 samples at two representative time steps during the transient. It is noted that the few-group parameter uncertainties propagated follow a normal distribution, however, the resulting core relative power distributions are not always normal. Shifting from normal distribution around the peak location indicates the noticeable presence of nonlinearity during the transient.

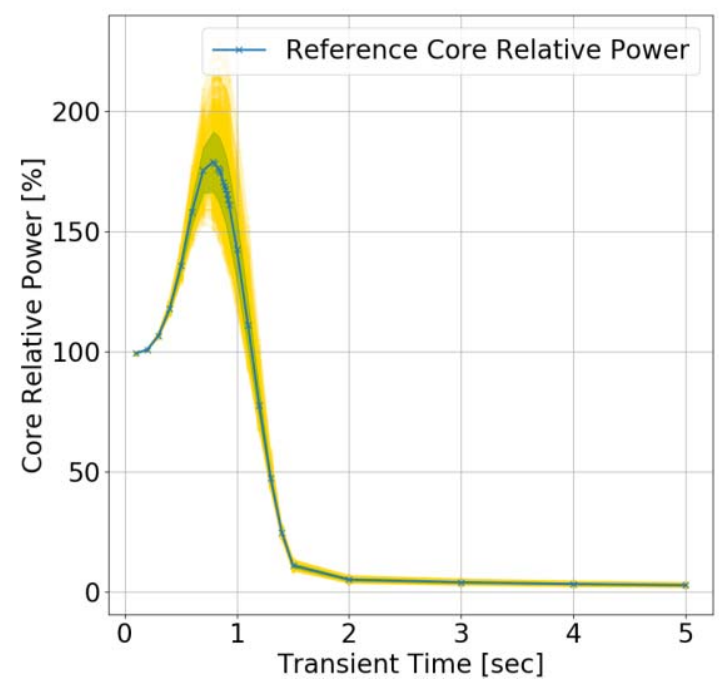


Figure 5. Uncertainty of NESTLE-C Core Power in a Loss of Coolant Accident Transient

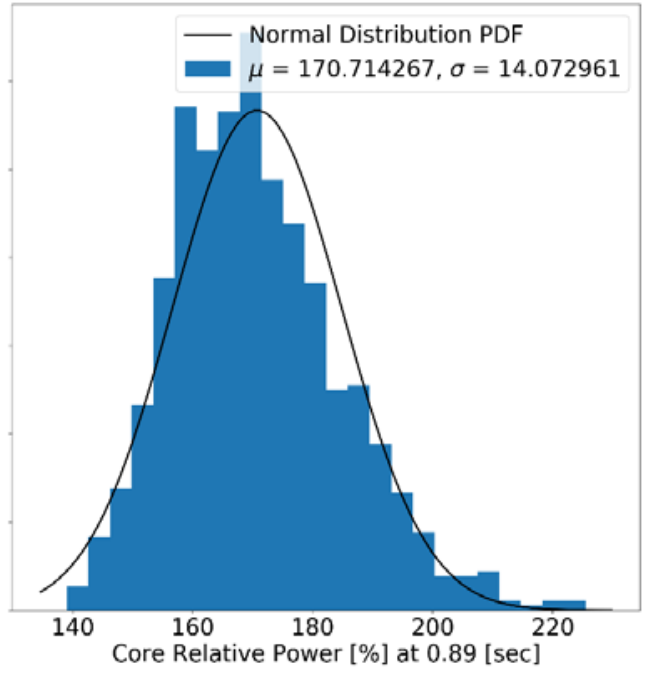

(a) Histogram of Core Power at Peak

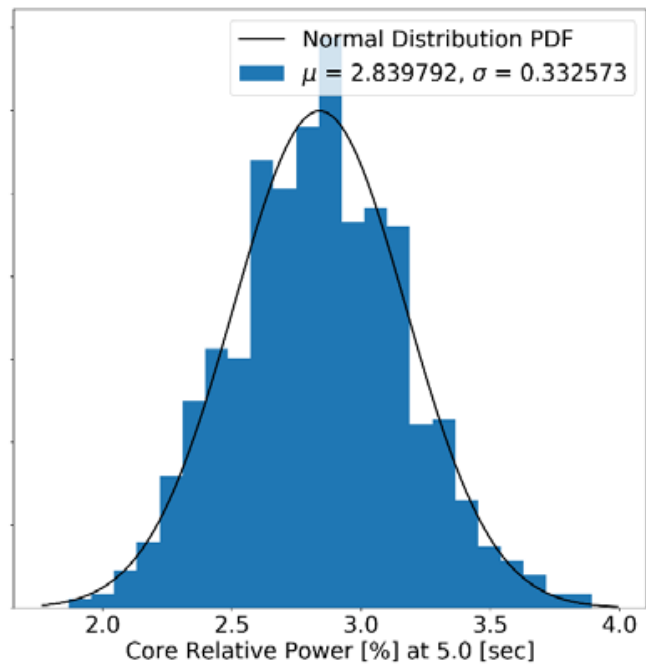

(b) Histogram of Core Power at End

Figure 6 Uncertainties of Core Power at Select Time Steps.

\section{MULTIGROUP TESTING}

SERPENT-based reference few-group parameters were prepared for NESTLE-C using two-, four-, and eight-group energy grids. The uncertainty libraries were correspondingly extended to include four-, and eight-group variants using the previously described ROM-based procedures. A test suite was developed using NESTLE-C to test the sensitivity of simulation results to the choice of group structure. Selected results are presented in Table 2 and Figure 7.

Table 2 presents the standard deviations for the neutron multiplication factors of a reference and "voided" core, along with the derived static coolant void reactivity worth, in mk. Results are listed based on the source of the reference cross section input. The SERPENT and HELIOS two-group cases shared the same uncertainty library data, while separate uncertainty libraries were developed for the SERPENT four- and eight-group cross section input data. Two- and four-group uncertainty data are based on a rank 30 reducedorder model, while the eight group uncertainty data are based on a rank 60 model. As can be seen, there is little sensitivity to the group structure.

Figure 7 compares the LOCA problem using different reference cross section and uncertainty data combinations. It is a broader generalization of Figure 5, which was limited to two-group data using the HELIOS reference cross section input data. The thick black solid curves represent the mean, or best estimate, of the core power evolution. The other curves serve to delimit the one- and two-sigma limits. As can be seen, there are only minor differences in results when SERPENT or HELIOS-based cross section inputs are used. Similarly, the results are broadly insensitive to the number of energy groups. 
Table 2

Standard deviations of NESTLE-C full core scalar responses

\begin{tabular}{|l|l|l|l|l|}
\hline \multicolumn{2}{|l}{ Response } & \multicolumn{4}{|c|}{ Standard deviations (mk) } \\
\hline & HELIOS 2G & SERPENT 2G & SERPENT 4G & SERPENT 8G \\
\hline Reference $\mathbf{k}_{\text {eff }}$ & 7.49 & 7.61 & 8.59 & 7.60 \\
\hline Voided core $\mathbf{k}_{\text {eff }}$ & 7.32 & 7.44 & 8.37 & 7.43 \\
\hline CVR (mk) & 0.46 & 0.46 & 0.56 & 0.51 \\
\hline
\end{tabular}
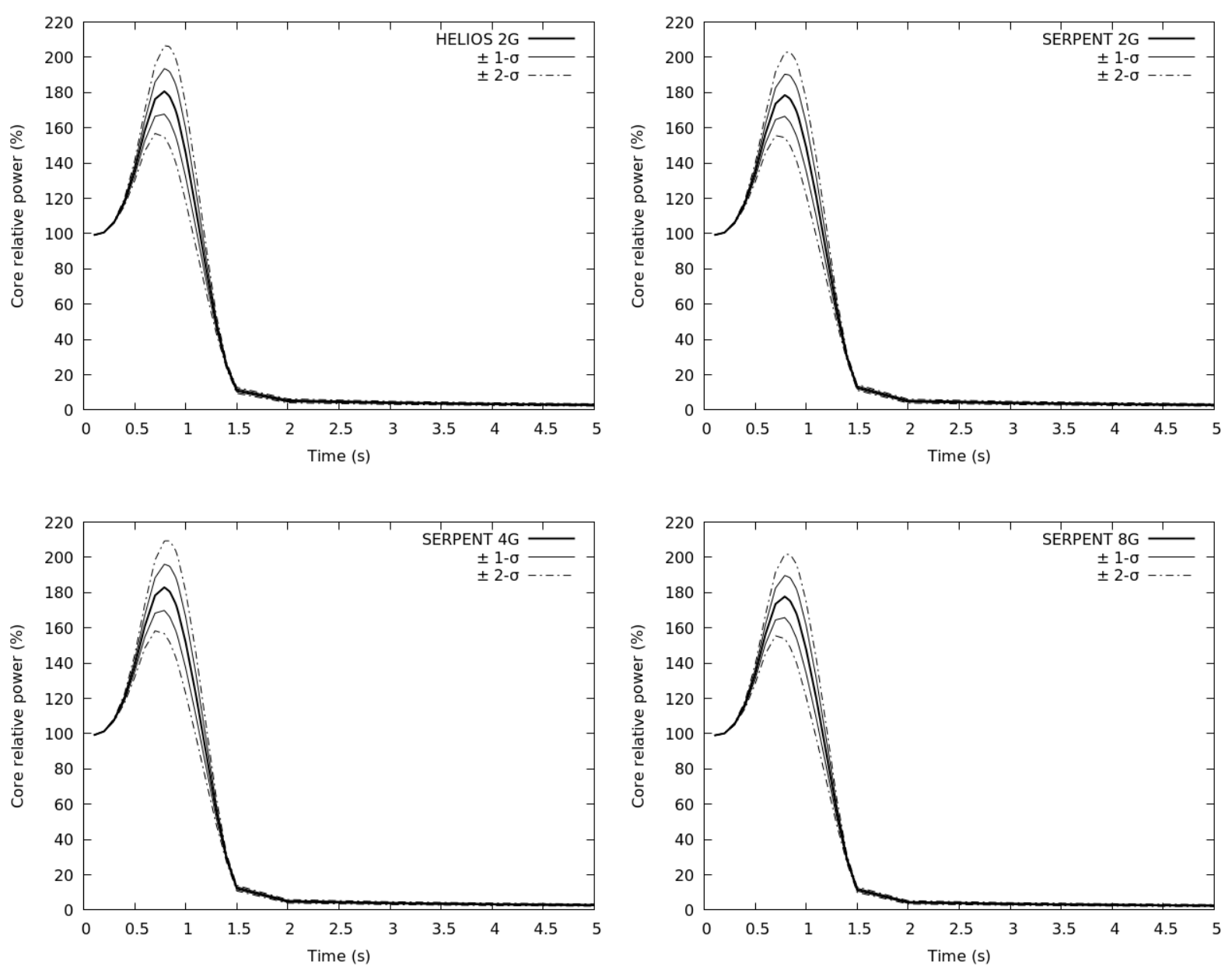

Figure 7. Uncertainty in the core relative power evolution of the LOCA test

\section{SUMMARY AND CONCLUSIONS}

Results of the first major milestone of an integrated uncertainty characterization framework for CANDU neutronics core simulation are reported in this paper. The goal was to develop an automated computational capability for the generation of an uncertainty library for steady state and transient full core CANDU 
simulations using the NESTLE-C core simulator. To our knowledge, this represents the first major effort to develop an uncertainty library for full core neutronics simulation, done in a manner that avoids simplifications, e.g., eliminating various local core dependencies, in favor of computational feasibility. Results indicate that the use of reduced order modeling allows for a seamless process for gaining computational advantage realized by a compression of the uncertainty space and done in a manner that can be mathematically defended. The impact of modeling assumptions was tested by exploring the sensitivity of the calculated uncertainties to modeling assumptions, e.g., use of Monte Carlo vs. deterministic models for radiation transport, or the use of continuous vs. multi-group cross-section representation. Future work will focus on sensitivity analysis, uncertainty mapping, and incorporating modeling uncertainties.

\section{REFERENCES}

1. Abdel-Khalik, H.S., "Feasibility study of an integrated framework for characterization of uncertainties with application to CANDU steady state and transient reactor physics simulation", Final Report RSP 598.1, June 2015, http://www.nuclearsafety.gc.ca/eng/pdfs/feasibility-study-integrated-framework.pdf

2. Serghiuta, D., Tholammakkil, J., Abdel-Khalik, H.S. and Trottier, A., 2017. Integrated Framework for Propagation of Uncertainties in Nuclear Cross-Sections in CANDU Steady-State and Transient Reactor Physics Simulations. 37th Annual Conference of the Canadian Nuclear Society, June 2017.

3. Serghiuta, D., Tholammakkil, J., and Abdel-Khalik, H.S., "BEPU and Evaluation of Predictive Capability of Physics Simulations of CANDU Transients", ANS Best Estimate Plus Uncertainty International Conference (BEPU 2018), Real Collegio, Lucca, Italy, May 13-19, 2018

4. Bang, Y., Abdel-Khalik, H. and Hite, J., "Hybrid reduced order modeling applied to nonlinear models," International Journal for Numerical Methods in Engineering, 91(9), pp.929-949, 2012.

5. Huang, D., Abdel-Khalik, H., Rabiti, C., and Gleicher, F., "Dimensionality Reducibility for MultiPhysics Reduced Order Modeling," Annals of Nuclear Energy, 110, December 2017.

6. Leppänen, J., et al., "The Serpent Monte Carlo code: Status, development and applications in 2013," Ann. Nucl. Energy, 82, 142-150, 2015.

7. Trottier, A., Pfeiffer, S., and Serghiuta, D., "SERPENT-based Few-Group Cross Sections Data for NESTLE-C", Proceedings of M\&C 2019, Portland, OR, August 25-29, 2019, Pages 1230-1238.

8. Turinsky, P. and Sarsour, H., "NESTLE-C: Few-Group Neutron Diffusion Equation Solver Utilizing the Nodal Expansion Method for Eigenvalue, Adjoint, Fixed-Source Steady-State and Transient Problems: CANDU Version", North Carolina State University, 2003.

9. DeHart, M., Jessee, M., "NEWT: a new transport algorithm for two-dimensional discrete ordinates analysis in non-orthogonal geometries," ORNL/TM-2005/39, 2005.

10. Williams, M., Ilas, G., Jessee, M., Rearden, B., Wiarda, D., Zwermann, W., Gallner, L., Klein, M., Krzykacz-Hausmann, B. and Pautz, A., "A statistical sampling method for uncertainty analysis with SCALE and XSUSA.” Nuclear Technology, 183, 3, 2013.

11. Pounders, J.M., Rahnema, F., Serghiuta, D. and Tholammakkil, J., 2011. A 3D stylized half-core CANDU benchmark problem. Annals of Nuclear Energy, 38(4), pp.876-896.

12. Trottier, A., Abdel-Khalik, H.S., "Multigroup Reference and Covariance Cross Section Data Libraries for NESTLE-C", Final Report, CNSC Research project R598.2, September 2019.

13. Huang, D. and Abdel-Khalik, H.S., (in press). "Theoretical development of cross-section uncertainty library for core simulators," ASME. ASME J of Nuclear Rad Sci., accepted for publication, August 2019

14. Huang, D. and Abdel-Khalik, H.S., (in press). "Application of cross sections uncertainty propagation framework to light and heavy water reactor systems," ASME. ASME J of Nuclear Rad Sci., accepted for publication, August 2019 